\title{
Optical and elastic properties of diamond-like carbon with metallic inclusions: A theoretical study
}

\section{Citation}

Tritsaris, Georgios A., Christos Mathioudakis, Pantelis C. Kelires, and Efthimios Kaxiras. 2012. "Optical and Elastic Properties of Diamond-Like Carbon with Metallic Inclusions: A Theoretical Study." Journal of Applied Physics 112 (10) (November 15): 103503. doi:10.1063/1.4765721.

\section{Published Version}

doi:10.1063/1.4765721

\section{Permanent link}

http://nrs.harvard.edu/urn-3:HUL.InstRepos:33371794

\section{Terms of Use}

This article was downloaded from Harvard University's DASH repository, and is made available under the terms and conditions applicable to Other Posted Material, as set forth at http:// nrs.harvard.edu/urn-3:HUL.InstRepos:dash.current.terms-of-use\#LAA

\section{Share Your Story}

The Harvard community has made this article openly available.

Please share how this access benefits you. Submit a story.

\section{Accessibility}




\title{
Optical and elastic properties of diamond-like carbon with metallic inclusions: A theoretical study
}

\author{
Georgios A. Tritsaris, ${ }^{1}$ Christos Mathioudakis, ${ }^{2}$ Pantelis C. Kelires, ${ }^{2}$ \\ and Efthimios Kaxiras ${ }^{1,3, a)}$ \\ ${ }^{1}$ School of Engineering and Applied Sciences, Harvard University, Cambridge, Massachusetts 02138, USA \\ ${ }^{2}$ Research Unit for Nanostructured Materials Systems, Department of Mechanical and Materials Science \\ Engineering, Cyprus University of Technology, P. O. Box 50329, 3603 Limassol, Cyprus \\ ${ }^{3}$ Department of Physics, Harvard University, Cambridge, Massachusetts 02138, USA
}

(Received 15 September 2012; accepted 17 October 2012; published online 19 November 2012)

\begin{abstract}
A tough material commonly used in coatings is diamond-like carbon (DLC), that is, amorphous carbon with content in four-fold coordinated $\mathrm{C}$ higher than $\sim 70 \%$, and its composites with metal inclusions. This study aims to offer useful guidelines for the design and development of metalcontaining DLC coatings for solar collectors, where the efficiency of the collector depends critically on the performance of the absorber coating. We use first-principles calculations based on density functional theory to study the structural, electronic, optical, and elastic properties of DLC and its composites with $\mathrm{Ag}$ and $\mathrm{Cu}$ inclusions at $1.5 \%$ and $3.0 \%$ atomic concentration, to evaluate their suitability for solar thermal energy harvesting. We find that with increasing metal concentration optical absorption is significantly enhanced while at the same time, the composite retains good mechanical strength: DLC with $70-80 \%$ content in four-fold coordinated C and small metal concentrations $(<3$ at. \%) will show high absorption in the visible (absorption coefficients higher than $10^{5} \mathrm{~cm}^{-1}$ ) and good mechanical strength (bulk and Young's modulus higher than 300 and $500 \mathrm{GPa}$, respectively). @ 2012 American Institute of Physics. [http://dx.doi.org/10.1063/1.4765721]
\end{abstract}

\section{INTRODUCTION}

Trough solar collectors are a technology for solar thermal energy conversion that can deliver emission-free solar power for on-site and centralized applications. ${ }^{1,2}$ The efficiency of the collector depends critically on the performance of the absorber coating. ${ }^{3-5}$ Materials based on amorphous carbon (a-C), including diamond-like carbon (DLC), that is, amorphous carbon with a content in four-fold coordinated $\mathrm{C}$ that is higher than $\sim 70 \%,{ }^{6,7}$ have been a favorable choice for absorbers because their optical and mechanical properties $^{7-12}$ can be tailored to satisfy the requirements of the intended application. For instance, the performance of a-C can be tuned with the incorporation of transition metal in the material, such as $\mathrm{Ag}, \mathrm{Cu}$, or $\mathrm{Ti}$ nanoparticles: $:^{11,13-21}$ for metal-containing DLC, the carbon matrix provides high mechanical strength and the metallic inclusion enhances optical absorption. The structure and properties of the composite generally depend on the preparation technique and the deposition conditions, ${ }^{6,8}$ which make the identification of universal structure-property relationships a challenging task..$^{10,22}$ Alongside with the current heightened interest in carbonbased materials, ${ }^{7,23}$ understanding how the structure of the metal-containing diamond-like carbon determines its properties is an important step towards the design and development of carbon-based, high-performance materials for solar thermal energy harvesting.

Theory and atomistic simulation have contributed significantly in clarifying how the macroscopic properties of materials based on a-C depend on the atomic structure. ${ }^{12,18,24-28}$

a)e-mail: kaxiras@physics.harvard.edu.
Studies of metal (Me)-containing DLC composites, in particular, are currently limited by the complexity of the structures involved and the computational challenges they present. ${ }^{18}$ Here, we use density functional theory (DFT) calculations to study the structural, electronic, optical, and elastic properties of model bulk structures of Me-DLC, namely, Ag-DLC and $\mathrm{Cu}$-DLC. The performance of the composites regarding their use as absorber coatings is evaluated with respect to optical efficiency and mechanical stability. Specifically, we calculate the absorption coefficient, as well as the bulk and Young's moduli, and we demonstrate how the properties of the composite depend on the atomic-scale features of the DLC/Me interface. We identify a correlation between these properties and the hybridization of $\mathrm{C}$. The findings suggest that with increasing metal concentration, optical absorption is significantly enhanced while at the same time, the composite retains good mechanical strength.

\section{MODELS AND METHODS}

We used amorphous bulk structures of 64 atoms to model metal-free DLC [Fig. 1(a)] and DLC composites with a content in $\mathrm{sp}^{3}$-bonded (four-fold coordinated) $\mathrm{C}$ in the range of $67-100 \%$ [Figs. 1(b)-1(d)]. For the metal-free DLC structures, the unit cell was prepared by quenching using the tight-binding method (more information is given in our previous work $^{12}$ ) and relaxed using standard DFT structural optimization by the minimization of all forces (to the upper limit of $0.05 \mathrm{eV}^{-1}$ ) and stresses (volume optimization). We further relaxed the structures using DFT-based molecular dynamics by first performing a simulation for $1.5 \mathrm{ps}$ in the microcanonical (N, V, E) ensemble (constant volume) using 
(a)

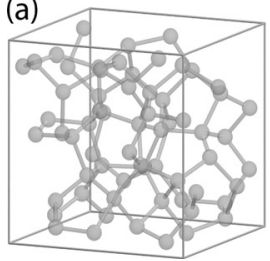

(b)

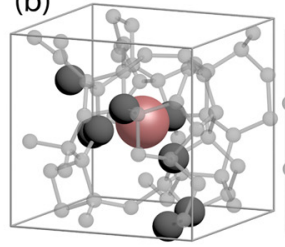

(c)

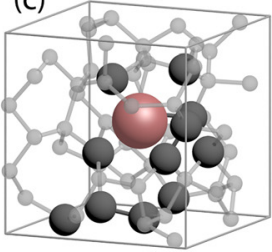

(d)

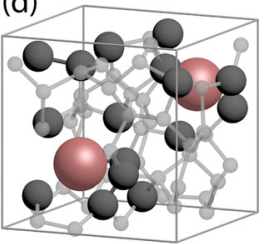

FIG. 1. Examples of model bulk structures of (a) metalfree and $[(\mathrm{b}),(\mathrm{c})$, and $(\mathrm{d})]$ metal $(\mathrm{Me})$-containing DLC: (a) $100 \% \mathrm{sp}^{3}$-bonded DLC, [(b) and (c)] 1.5 at. \% Me-DLC and (d) 3.0 at. \% Me-DLC. Gray-, black- and red-shaded atoms represent $\mathrm{sp}^{3}$-bonded (four-fold coordinated) $\mathrm{C}, \mathrm{sp}^{2}$-bonded (three-fold coordinated) $\mathrm{C}$, and metallic inclusions, respectively. a temperature of $800 \mathrm{~K}$ and then by quenching the structure to $0 \mathrm{~K}$ in steps of $0.5 \mathrm{~K} \mathrm{fs}^{-1}$. After the molecular dynamics simulation, the atomic positions were relaxed. A fully $\mathrm{sp}^{3}-$ bonded DLC, which in the following we refer to as "amorphous diamond" (a-D, density $3.4 \mathrm{~g} / \mathrm{cm}^{-3}$ ), was represented by a continuous random network model, ${ }^{29}$ which, although it corresponds to an idealized case, ${ }^{30}$ we use as a reference structure [Fig. 1(a)]. We substituted one or two $\mathrm{sp}^{3}$-bonded $\mathrm{C}$ atoms with $\mathrm{Ag}, \mathrm{Cu}$, or $\mathrm{Al}$, to model DLC composites with $1.5 \%$ [Figs. 1(b) and 1(c)] and 3.0\% [Fig. 1(d)] metal content (low concentration limit), and we relaxed the composite structure with molecular dynamics/structural optimization in the same way as for metal-free DLC. The volume of the unit cell was not optimized further. Volume relaxation could lead to lower content in $\mathrm{sp}^{3}$-bonded $\mathrm{C}^{27}$ but in the limit of single-atom metallic inclusions we expect the breaking of $\mathrm{C}-\mathrm{C}$ bonds with the introduction of metal atoms in the DLC to dominate the graphitization of the material. We note in passing that although hydrogenated DLC is also used in similar applications as hydrogen-free DLC, ${ }^{6,8}$ here we calculate only hydrogen-free structures.

For molecular dynamics and structural optimization, we used the SIESTA implementation ${ }^{31}$ of DFT (linear combination of numerical atomic orbitals, norm-conserving pseudopotential approach). A double- $\zeta$ with polarization basis set was used to represent the Kohn-Sham orbitals. For the description of exchange and correlation (xc), the PBEsol functional $^{32}$ was chosen because it typically yields good equilibrium properties, such as lattice constants and elastic moduli. The study of electronic and optical properties relied on the GPAW code, ${ }^{33}$ a grid-based approach based on projected augmented-wave method. ${ }^{34}$ A Monkhorst-Pack mesh of $2 \times 2 \times 2$ special points was used for integration in $\mathrm{k}$ space. Dielectric functions were calculated within the framework of time-dependent DFT (TDDFT) ${ }^{35}$ using the bootstrap approach of Sharma et al., ${ }^{36}$ which is based on an approximate expression for the xc kernel and its numerical derivation. The scheme has been demonstrated to give optical spectra in good agreement with experiment for prototypical structures with small computational effort. ${ }^{36,37}$ For the calculation of dielectric functions, empty electronic bands up to $40 \mathrm{eV}$ above the Fermi level were included and a smearing factor of $0.40 \mathrm{eV}$ was used to broaden the spectra. The calculated dielectric functions can be only as accurate as the electronic structure of the ground state. We used the GLLBSC $\mathrm{xc}$ functional introduced by Kuisma et al. ${ }^{38}$ (orbital-dependent functional based on the potential of Gritsenko et al., ${ }^{39}$ with elements from PBEsol) to improve the description of the noble metal electronic states. ${ }^{40,41}$ A finite deformation (stress-strain) approach was used for the numerical derivation of elastic matrices from which the bulk and Young's moduli were calculated. ${ }^{42}$ SIESTA and GPAW were used via the Atomic Simulation Environment (ASE) interface. ${ }^{43}$

\section{RESULTS AND DISCUSSION}

The first step in studying the properties of Me-DLC is to understand the effect of the metallic inclusion on the bonding of $\mathrm{C}$ in the composite. Experiment has demonstrated that increasing metal concentration in Me-DLC induces the progressive graphitization of the carbon matrix. ${ }^{18,44}$ Me-DLC films are prepared with metal nano-inclusions whose mean size is typically $<10 \AA .{ }^{17,18}$ To relate our theoretical study to experiment, we examine the nature of the DLC/Me interface. Fig. 1 shows that an interface exists in the Me-DLC composites that encapsulates the metal atom and resembles a uniform spherical shell: The interface is rich in $\mathrm{sp}^{2}$-bonded $\mathrm{C}$ because the substitution of $\mathrm{C}$ atoms in DLC with metal atoms removes multiple $\mathrm{C}-\mathrm{C}$ bonds. In other words, incorporation of metal atoms in the DLC changes the hybridization of C. To quantify the graphitization of the carbon matrix, we calculated the content in $\mathrm{sp}^{3}$-bonded $\mathrm{C}$ for metal-free and metal-containing DLC structures. We used the C-C pair distribution function to calculate the cut-off distance for the first nearest neighbors of $\mathrm{C}$ in the structure, and with this cut-off distance, the number of nearest neighbors of each $\mathrm{C}$ atom was calculated. ${ }^{12}$ In Fig. 2, we show how the content in fourfold coordinated C of DLC is reduced when metal atoms are incorporated in the material. The progressive graphitization of the DLC matrix expressed by $\Delta \mathrm{sp}^{3}$ with increasing metal concentration $[\mathrm{Me}$ ] for our structural models is described by the linear relationship $\Delta \mathrm{sp}^{3}=-6.9[\mathrm{Me}]$. The trend in graphitization (or equivalently, the slope of the linear equation: -6.9) depends on the distribution of the metallic inclusions. For example, we would expect a different trend (that is, a less steep slope), if the metallic inclusions were clustered instead of well dispersed in the host material. Our

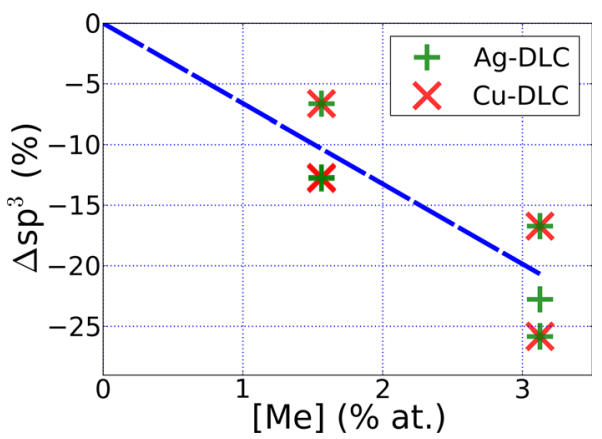

FIG. 2. Change in the $\mathrm{sp}^{3}$-bonded $\mathrm{C}, \Delta \mathrm{sp}^{3}$, as a function of the metal concentration $[\mathrm{Me}]$ in $\mathrm{Ag}$ - (green) and $\mathrm{Cu}$ - (red) containing DLC. The slope of the approximate linear fit to the results corresponds to $6.9 \%$ decrease in $\mathrm{sp}^{3}$ bonded $\mathrm{C}$ for $1 \%$ increase in metal content. 
model follows closely the trend observed in experiment ${ }^{18}$ where Ag-DLC composites of Ag concentrations up to $~ 8 \%$ were prepared with pulsed laser deposition, and the observed result is a decrease of $\sim 8.5 \%$ in $\mathrm{sp}^{3}$-bonded $\mathrm{C}$ for 1 at. $\%$ increase in metal content. Nevertheless, for small metal concentrations, the DLC maintains its highly $\mathrm{sp}^{3}$-bonded character. For example, for pure DLC with $85 \% \mathrm{sp}^{3}$-bonded C, incorporation of $3 \% \mathrm{Ag}$, will result in a composite with $\sim 65 \% \mathrm{sp}^{3}$-bonded $\mathrm{C}$, that is, a $\sim 20 \%$ reduction. Moreover, Fig. 2 suggests that the carbon matrix will be fully graphitized at metal concentrations of $8-12 \%$. This limit is consistent with experiment, ${ }^{18,44}$ but any extrapolation out of the range of the calculated results should be treated with caution. We note that the trends in the graphitization for our model DLC composites with $\mathrm{Ag}$ and $\mathrm{Cu}$ are indistinguishable from each other (see Fig. 2). Larger structures may be needed to reveal finer differences in the hybridization of $\mathrm{C}$ between composites with different metals.

Up to this point, we have established that incorporation of metal atoms in DLC induces the graphitization of the carbon matrix. We proceed to study the effect of the metal on the electronic structure, which essentially determines the optical absorption of the material. The density of states (DOS) of a-D and the DOS of a Ag-containing (1.5 at. \%) DLC composite are juxtaposed in Fig. 3. For a-C, the optical band gap, as well as the mobility gap (that is, the separation energy between extended and localized electronic states), is higher than the Kohn-Sham band gap due to bonding disorder. Still, in the case of a-D, it is expected that the corresponding band gaps are comparable because a-D is fully $\mathrm{sp}^{3}$ bonded with relatively limited bonding disorder. We calculate the energy difference between the highest occupied and the lower unoccupied Kohn-Sham states for a-D to be $4.15 \mathrm{eV}$. DFT calculations based on the (semi-)local approach for xc, which do not account for the derivative discontinuity of the xc potential, typically underestimate the fundamental band gap. For amorphous structures, the band gap is expected to be overestimated due to the finite size of our structural models. The calculated Kohn-Sham gap is in reasonable agreement with experiment ${ }^{45}$ where the optical
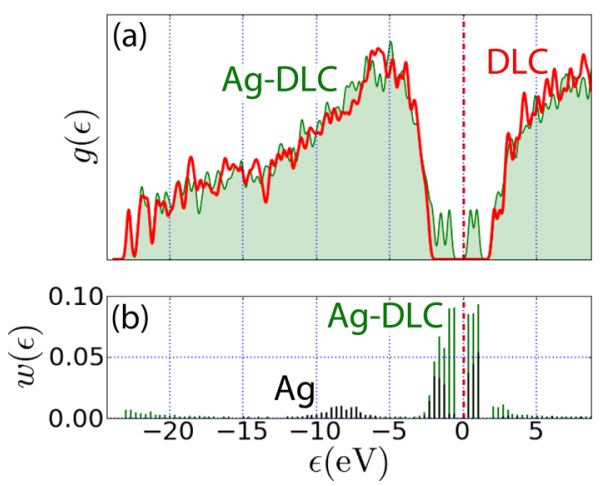

FIG. 3. (a) Density of states $g(\epsilon)$ of a fully $\mathrm{sp}^{3}$-bonded (red curve), and $83 \%$ $\mathrm{sp}^{3}$-bonded, Ag-containing (1.5 at. \%) diamond-like carbon (green, shaded curve). (b) Degree $w(\epsilon)$ of electronic state localization. Localization of $w(\epsilon) \rightarrow 0$ signifies an extended wavefunction, whereas $w(\epsilon)=1$ corresponds to a wavefunction that is localized on a single atom. The black and green lines correspond to all atoms and $\mathrm{Ag}$ only. The red dashed line marks the middle of the gap. band gap of an essentially four-fold coordinated DLC is reported to be up to $4.5 \mathrm{eV}$ and tight-binding calculations ${ }^{26}$ where a band gap of $5 \mathrm{eV}$ is reported. We attribute this agreement to a cancelation between the errors introduced by the $\mathrm{xc}$ and the structural models we use. These results should be contrasted to the case of crystalline diamond for which the experimental band gap is $5.5 \mathrm{eV}$.

In contrast to the case of a-D where a clean Kohn-Sham band gap exists, when metal atoms are incorporated in DLC, electronic states are introduced in the band gap [Fig. 3(a)]. The degree of localization, $w$, for the electronic states of a 1.5 at. \% Ag-83\% sp ${ }^{3}$ DLC composite is shown in Fig. 3(b). We define and calculate $w$ as follows:

$$
w(\epsilon)=\sum\left(N g_{i}(\epsilon) / g(\epsilon)-1\right)^{2} / N(N-1) \text { for } g(\epsilon)>0,
$$

where $g_{i}(\epsilon)$ is the density of states projected on an atom "i" of the composite and $N$ is the total number of atoms in the structure ( $N=64$ for our structural models). The sum is taken over all atoms. The calculation of $w(\epsilon)$ corresponds to a participation ratio analysis: for an electronic state with energy $\epsilon$ that is extended $g_{i}(\epsilon) \rightarrow g(\epsilon) / N$ and $w(\epsilon) \rightarrow 0$. On the other hand, for an electronic state that is localized at a single atom "i" $g_{i}(\epsilon)=g(\epsilon)$ and $g_{j}(\epsilon)=0$ for $i \neq j$, thus $w(\epsilon)=1$. Inspection of Fig. 3(b) indicates that the metallic inclusion introduces states in the band gap that are localized, arising from both the metal itself and the bonding disorder it induces in the carbon matrix. Moreover, the states in the energy range between -10 and $-5 \mathrm{eV}$ (energies being given with respect to the middle of the band gap of a-D) are also relatively more localized as they partly describe the $d$ electronic states of $\mathrm{Ag}$.

In general, the mobility of the carriers in DLC is low (in the range of $10^{-12}-10^{-11} \mathrm{~cm}^{2} \mathrm{~V}^{-1}$ ) and values in the range of $10^{2}-10^{16} \Omega \mathrm{cm}$ have been reported for the resistivity of DLC depending on preparation conditions. ${ }^{9}$ Incorporation of metallic inclusions in a-C can increase the conductivity. ${ }^{9,16}$ As far as the electronic structure is concerned (see Fig. 3), the band tails are determined mainly from (valence) $\pi$ and (conduction) $\pi *$ states that arise from $\mathrm{sp}^{2}$-bonded C. ${ }^{7,26,46}$ With increasing metal content (increasing content in $\mathrm{sp}^{2}$ bonded $\mathrm{C}$ ), the $\pi(*)$ bands broaden and the band tails become wider. ${ }^{9,26}$ However, for small metal concentrations, the shape of the DOS of Me-DLC is not substantially different from that of metal-free DLC (Fig. 3). Moreover, the electronic states in the band gap are localized. Thus, we do not expect incorporation of metal atoms in DLC at the low concentration limit to enhance conductance enough to render Me-DLC composites active components, for example, in photovoltaic applications.

Despite these expectations, in the following, we show that Me-DLC composites can be key components for passive applications, for example, as optical absorbers for solar thermal collectors. In general, the efficiency of the collector depends on the spectral selectivity of the absorber film. The ratio of solar radiation absorbed to the thermal radiation emitted defines the selectivity of the material. An efficient absorber is expected to show large absorption at wavelengths below $2 \mu \mathrm{m}$ and low absorption at wavelengths higher 

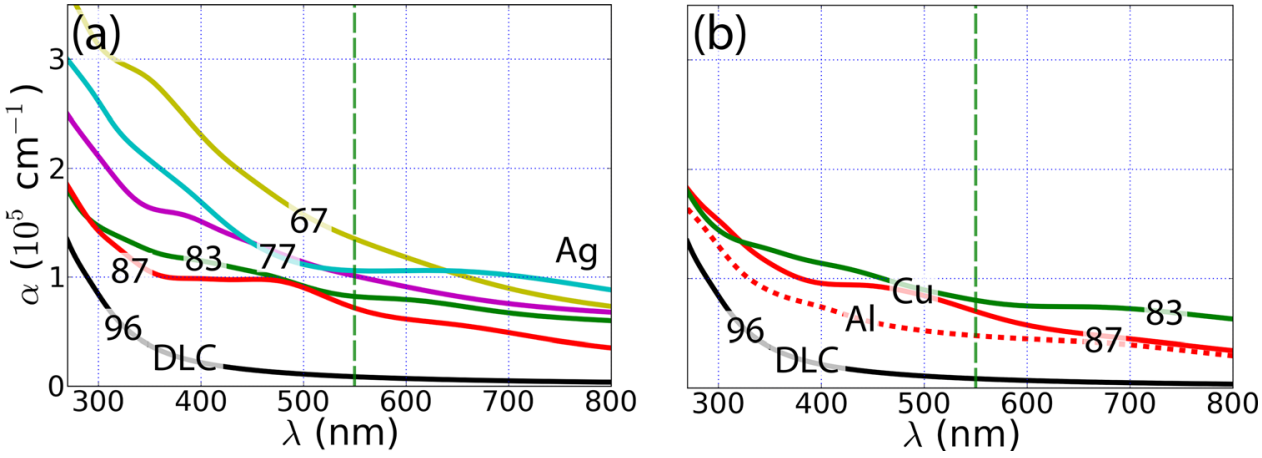

FIG. 4. Absorption coefficient $\alpha$ for metal-free (black line), and Al- (dashed line), $\mathrm{Cu}$ - and Ag- (solid lines) containing DLC. Different colors correspond to different content in $\mathrm{sp}^{3}$-bonded $\mathrm{C}$ (indicated as percentage on top of each family of curves) and metal. The peak of the solar spectrum $(\lambda=550 \mathrm{~nm})$ is marked with a vertical green, dashed line. than $2 \mu \mathrm{m}$. A property of the material that describes the amount of light absorbed is the absorption coefficient $\alpha$. In Fig. 4, we show how $\alpha$ depends on the wavelength $\lambda$ for MeDLC of various contents in $\mathrm{sp}^{3}$-bonded $\mathrm{C}$ and metal, and for wavelengths in the visible range of the solar spectrum ( $\lambda \simeq 400-700 \mathrm{~nm})$. We calculate the absorption coefficient as $\alpha(E)=E \varepsilon_{2}(E) / n(E)$, where $n$ is the refractive index, calculated from $n(E)=\left(\varepsilon_{1}(E)+\sqrt{\varepsilon_{1}(E)^{2}+\varepsilon_{2}(E)^{2}}\right) / 2$, with the real and imaginary part of the dielectric function denoted by $\varepsilon_{1}$ and $\varepsilon_{2}$.

The calculation of the dielectric function of the composites can only be as accurate as the description of the electronic structure. For Ag, the $d$ electronic states are typically calculated with standard DFT to lie higher in energy in comparison to experiment. ${ }^{40,41} \mathrm{We}$ used the orbital-dependent

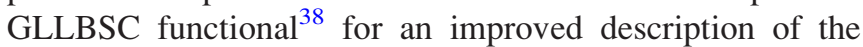
electronic structure. To assess the validity of our method, we calculated the bulk plasmon energy for Ag with both LDA and GLLBSC from the electron energy loss functions ${ }^{35}$ obtained at wavevectors in the range of $0.04-0.20 \AA^{-1}$. For these calculations, we used the adiabatic local density approximation for the xc kernel, and we sampled the Brillouin zone with a $70 \times 70 \times 70$ Monkhorst-Pack grid. We find the plasmon energy to be $3.0 \mathrm{eV}$ from LDA, and $3.5 \mathrm{eV}$ from GLLBSC calculations. The GLLBSC energy lies closer to the measured energy of $3.83 \mathrm{eV}^{47}$ in comparison to the energy obtained with LDA as the relative positions in energy of the $\mathrm{Ag} d$ - and $s p$-levels are described more accurately with GLLBSC, and in agreement with previous theoretical work. ${ }^{40}$ For instance, for the $1.5 \% \mathrm{Ag}-83 \% \mathrm{sp}^{3}$ DLC, we calculate with GLLBSC the peak of the $d$ states to be $0.5 \mathrm{eV}$ lower in energy compared to the LDA calculation.

From the dielectric function, we calculated the absorption coefficient and in Table I we give its value $\alpha_{550}$ at the wavelength $\lambda=550 \mathrm{~nm}$, which we use as a figure of merit for absorption efficiency at wavelengths near the peak of the solar spectrum. For example, we calculate the optical absorption for a metal-free, $96 \% \mathrm{sp}^{3}$-bonded DLC structure to be $\alpha_{550}=0.9 \times 10^{4} \mathrm{~cm}^{-1}$. In contrast, incorporation of $\mathrm{Ag}$ or $\mathrm{Cu}$ in DLC enhances optical absorption at short wavelengths $(\lambda<550 \mathrm{~nm})$ significantly, whereas the descending tail of $\alpha$ at higher wavelengths is less affected. For example, we calculate $\alpha_{550} \simeq 7 \times 10^{4} \mathrm{~cm}^{-1}$ for composites of 1.5 at. $\%$ $\mathrm{Ag}$ - and $\mathrm{Cu}-87 \%$ DLC. For both the metal-free and metalcontaining DLC, optical transitions between extended to extended, localized to extended, and extended to localized electronic states contribute to the absorption. As mentioned previously, the optical band gap of an essentially four-fold coordinated DLC is in the range of $4.5-5 \mathrm{eV}$, which corresponds to the relatively short absorption wavelength of $\sim 1240 / 5.0 \mathrm{~nm}=250 \mathrm{~nm}$. In our structural models, the incorporation of metal atoms in DLC affects optical absorption mostly indirectly, via the DLC/Me interface, which is rich in three-fold coordinated $\mathrm{C}$, as there is almost no difference between the optical response of $\mathrm{Cu} \mathrm{Ag}$ - and $\mathrm{Cu}$-containing DLC (Fig. 4). With progressive graphitization, the bands broaden, the optical band gap becomes narrower, and optical absorption increases (see also Table I).

In general, the spatial distribution of the three-fold coordinated $\mathrm{C}$ affects the optical properties of a-C. DLC films with the same content in $\mathrm{sp}^{3}$-bonded $\mathrm{C}$ but different clustering of $\mathrm{sp}^{2}$-bonded $\mathrm{C}$ will exhibit different electronic and optical properties. ${ }^{6,26}$ However, in all our model structures, the configuration of the DLC/Me interface is similar (shell-like), which suggests that the concentration of $\mathrm{sp}^{3}$-bonded $\mathrm{C}$ may be used as a single descriptor for the absorption of Me-DLC composites, without the need to consider the clustering of the three-fold coordinated C. Indeed, we find a correlation between the absorption coefficient $\alpha_{550}$ and the content in $\mathrm{sp}^{3}$-bonded $\mathrm{C}$ described approximately by the linear equation $\alpha_{550}=-3.3 \times 10^{3} \mathrm{sp}^{3}+3.6 \times 10^{5}$, for concentration $\mathrm{sp}^{3}$ of $\mathrm{sp}^{3}$-bonded $\mathrm{C} \mathrm{sp}^{3}>0.67(67 \%$ is the lowest content in fourfold coordinated $\mathrm{C}$ in our set of structures, see also Table I).

To better understand the effect of transition metal on the optical absorption of Me-DLC, we modeled a $87 \% \mathrm{sp}^{3}$ bonded DLC composite with 1.5 at. \% Al, which has no $d$

TABLE I. Absorption coefficient $\alpha_{550}$ of metal-free $([\mathrm{Me}]=0)$ and Al-, $\mathrm{Cu}$ and Ag-containing $([\mathrm{Me}]=1.5,3.0$ at. \%) diamond-like carbon for the wavelength of $550 \mathrm{~nm}$.

\begin{tabular}{lcc}
\hline \hline $\mathrm{sp}^{3}$-bonded C (\%) & {$[\mathrm{Me}]($ at. $\%)$} & $\alpha_{550} \times 10^{4}$ \\
\hline 100 & 0 & 0.7 \\
96 & 0 & 0.9 \\
87 & $1.5 \mathrm{Al}$ & 4.8 \\
87 & $1.5 \mathrm{Cu}$ & 7.0 \\
87 & $1.5 \mathrm{Ag}$ & 7.1 \\
83 & $1.5 \mathrm{Cu}$ & 8.0 \\
83 & $1.5 \mathrm{Ag}$ & 8.2 \\
77 & $1.5 \mathrm{Ag}$ & 10.6 \\
77 & $3.0 \mathrm{Ag}$ & 10.0 \\
67 & $3.0 \mathrm{Ag}$ & 13.5 \\
\hline \hline
\end{tabular}



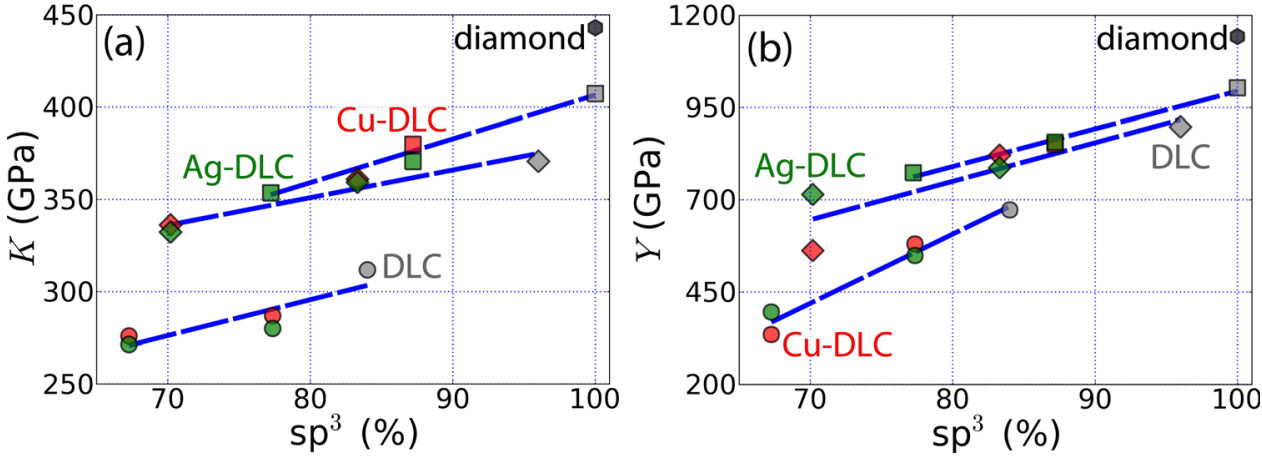

FIG. 5. (a) Bulk $K$ and (b) Young's $Y$ modulus of metal-free (gray), and Ag(red) and $\mathrm{Cu}$ - (green) containing DLC. Different symbols mark different content in $\mathrm{sp}^{3}$-bonded $\mathrm{C}$ in the unmodified (metal-free) DLC. A linear relationship describes the correlation between the content in $\mathrm{sp}^{3}$-bonded $\mathrm{C}$ and the elastic moduli. electrons. For the same concentration of $\mathrm{sp}^{3}$-bonded $\mathrm{C}$ and metal, we calculate lower absorption at shorter wavelengths for the Al-containing DLC compared to the $\mathrm{Ag}$ - and $\mathrm{Cu}$ containing DLC with $\alpha_{550}=4.8 \times 10^{4}$ for Al-DLC compared to $\alpha_{550}=7.0 \times 10^{4}$ for Ag- and Cu-DLC (see also Fig. 4). Finally, surface plasmon resonance can enhance the optical absorption of transition metal-containing $\mathrm{DLC}^{17,48}$ but our structural model (single-atom metallic inclusions) does not allow for the study of these collective excitations.

Lastly, we address the issue of the effect of metallic inclusions on the mechanical properties of the composite structures. Mechanical strength is necessary for the absorber coating to be stable under environmental conditions, but in $\mathrm{a}-\mathrm{C}$ the higher the content in three-fold coordinated $\mathrm{C}$, the less hard the material is. ${ }^{7,8,12}$ Thus, the metallic inclusions have beneficial effects on absorption but may have deleterious effects on mechanical strength. We calculated the bulk $K$ and Young's $Y$ moduli to evaluate the mechanical strength of the model structures (Fig. 5). The moduli for each structure were calculated from the $6 \times 6$ elastic matrix $c_{\mathrm{ij}}$ (in Voigt notation), which was derived using compression/elongation up to $0.2 \%$ and shear deformations of the unit cell. The bulk modulus $K$ of the DLC structures was calculated from the expression $9 K=\sum_{1<\mathrm{i}<3} c_{\mathrm{ii}}+2 \sum_{1<\mathrm{i}<\mathrm{j}<3} c_{\mathrm{ij}}$ and $Y$ from $Y=9 K G /$ $(3 K+G)$, where $G$ is the shear modulus obtained from $15 G$ $=\sum_{1<\mathrm{i}<3} c_{\mathrm{ii}}+3 \sum_{4<\mathrm{i}<6} c_{\mathrm{ii}}-\sum_{1<\mathrm{i}<\mathrm{j}<3} c_{\mathrm{ij}}$. For the cubic diamond crystal, we calculate $K=440 \mathrm{GPa}$ and $Y=1154 \mathrm{GPa}$ $\left(c_{11}=1010 \mathrm{GPa}, c_{12}=155 \mathrm{GPa}, c_{44}=621 \mathrm{GPa}\right)$. The calculated moduli are within $1 \%$ error compared to experiment, where the measured moduli are $K=443 \mathrm{GPa}$ and $Y=1145$ GPa. For a-D, we calculate $K=407 \mathrm{GPa}$ and $Y=1003 \mathrm{GPa}$, that is, $10 \%$ less than in the case of crystalline diamond. Moreover, we calculate $K=370 \mathrm{GPa}$ and $311 \mathrm{GPa}$ for metal-free DLC with $96 \%$ and $83 \%$ content in $\mathrm{sp}^{3}$-bonded C, respectively. The trends are in agreement with our earlier calculations based on the tight-binding method ${ }^{12,24}$ although the moduli differ within a maximum error of $10 \%$. We attribute the discrepancy to the increased accuracy offered by DFT: for crystalline diamond, Refs. 12 and 24 report $K=428 \mathrm{GPa}$ and $480 \mathrm{GPa}$, respectively. For the metal-containing DLC, we find that $K$ is reduced, on average, by $20 \mathrm{GPa}$ and $Y$ by $130 \mathrm{GPa}$ for every $10 \%$ decrease in $\mathrm{sp}^{3}$-bonded $\mathrm{C}$ (see Fig. 5). We stress that the linear relationship is approximate and may not hold for highly graphitized a-C. ${ }^{12}$ Nevertheless, by comparing between metal-free with metal-containing DLC with the same content in $\mathrm{sp}^{3}$-bonded $\mathrm{C}$, it is possible for the latter to have higher elastic moduli than the moduli of other, unmodified structures. Overall, Fig. 5 demonstrates that the mechanical strength of DLC is high enough for practical applications even after metal has been incorporated in the material. Our findings suggest that within the region of highly $\mathrm{sp}^{3}$-bonded a-C, a DLC material with 70-80\% content in four-fold coordinated C and small metal concentrations $(<3$ at. \%) will show high absorption in the visible $\left(\alpha>10^{5} \mathrm{~cm}^{-1}\right)$ and good mechanical strength $(K>300 \mathrm{GPa}, Y>500 \mathrm{GPa})$.

\section{CONCLUSIONS}

What is then the optimal composition of a highperformance metal-containing DLC composite for use as absorber coating for solar thermal energy conversion? Our findings suggest that there is no simple answer to this question. The performance of the absorber material is a compromise between its optical efficiency and mechanical stability: The change in $\mathrm{sp}^{3}$-bonded $\mathrm{C}$ is controlled by the amount of metal content, with an approximate linear relationship of $7 \%$ decrease for $1 \%$ increase in metal content. The progressive graphitization of the DLC matrix that is induced by increasing metal concentration in the composite enhances optical absorption in the visible. At the same time, incorporation of metal atoms softens the material. Nevertheless, the composite retains good mechanical strength. The two structureproperty relationships offered by our study can serve as guidelines for the design and development of metalcontaining diamond-like carbon coatings for solar thermal energy harvesting, by choosing the right metal content to achieve the desired behavior.

\section{ACKNOWLEDGMENTS}

G. T. wishes to thank Panagiotis Patsalas and Jun Yan for the helpful discussions. Financial support from the Strategic Infrastructure Project NEW INFRASTRUCTURE/ $\Sigma$ TPATH/ 0308/04 of DESMI 2008, which is co-financed by the European Regional Development Fund, the European Social Fund, the Cohesion Fund, and the Research Promotion Foundation of the Republic of Cyprus, is greatly acknowledged. Computations were performed on the Odyssey cluster at Harvard University, supported by the FAS Science Division Research Computing Group, and the SEAS HPC cluster, supported by the Academic Computing Group.

\footnotetext{
${ }^{1}$ N. Armaroli and V. Balzani, Angew. Chem., Int. Ed. 46, 52-66 (2007).

${ }^{2}$ M. Romero and A. Steinfeld, Energy Environ. Sci. 5, 9234 (2012).

${ }^{3}$ P. Oelhafen and A. Schüler, Sol. Energy 79, 110 (2005).
} 
${ }^{4}$ C. M. Lampert, Sol. Energy Mater. 1, 319 (1979).

${ }^{5}$ B. Seraphin, in Solar Energy Conversion, edited by B. Seraphin (Springer Berlin, Heidelberg, 1979), pp. 5-55.

${ }^{6}$ Y. Lifshitz, Diamond Relat. Mater. 8, 1659 (1999).

${ }^{7}$ J. Robertson, Mater. Sci. Eng. R 37, 129 (2002).

${ }^{8}$ A. Erdemir and C. Donnet, J. Phys. Chem. D 39, R311 (2006).

${ }^{9}$ A. Grill, Thin Solid Films 355-356, 189 (1999).

${ }^{10}$ Y. Lifshitz, Diamond Relat. Mater. 6, 687 (1997).

${ }^{11}$ G. Matenoglou, G. A. Evangelakis, C. Kosmidis, S. Foulias, D. Papadimitriou, and P. Patsalas, Appl. Surf. Sci. 253, 8155 (2007).

${ }^{12}$ C. Mathioudakis, G. Kopidakis, P. C. Kelires, C. Z. Wang, and K. M. Ho, Phys. Rev. B 70, 125202 (2004).

${ }^{13}$ K. Chiba, T. Takahashi, T. Kageyama, and H. Oda, Appl. Surf. Sci. 246, 48 (2005).

${ }^{14}$ C. Corbella, E. Bertran, M. C. Polo, E. Pascual, and J. L. Andújar, Diamond Relat. Mater. 16, 1828 (2007).

${ }^{15}$ W. Dai, P. Ke, M.-W. Moon, K.-R. Lee, and A. Wang, Thin Solid Films 520, 6057 (2012).

${ }^{16}$ J. L. Endrino, D. Horwat, R. Gago, J. Andersson, Y. S. Liu, J. Guo, and A. Anders, Solid State Sci. 11, 1742 (2009).

${ }^{17}$ S. Hussain, R. Roy, and A. Pal, Mater. Chem. Phys. 99, 375 (2006).

${ }^{18}$ G. M. Matenoglou, H. Zoubos, A. Lotsari, C. E. Lekka, P. Komninou, G. P. Dimitrakopulos, C. Kosmidis, G. A. Evangelakis, and P. Patsalas, Thin Solid Films 518, 1508 (2009).

${ }^{19}$ R. Paul, S. R. Bhattacharyya, R. Bhar, and A. K. Pal, Appl. Surf. Sci. 257, 10451 (2011).

${ }^{20}$ H.-S. Zhang, Appl. Surf. Sci. 255, 2551 (2008).

${ }^{21}$ J. Patscheider, T. Zehnder, and M. Diserens, Surf. Coat. Technol. 146147, 201 (2001).

${ }^{22}$ P. Patsalas, S. Logothetidis, and P. C. Kelires, Diamond Relat. Mater. 14, 1241 (2005).

${ }^{23}$ A. H. Castro Neto, F. Guinea, N. M. R. Peres, K. S. Novoselov, and A. K. Geim, Rev. Mod. Phys. 81, 109 (2009).

${ }^{24}$ M. G. Fyta, I. N. Remediakis, P. C. Kelires, and D. A. Papaconstantopoulos, Phys. Rev. Lett. 96, 185503 (2006)

${ }^{25}$ N. A. Marks, D. R. McKenzie, B. A. Pailthorpe, M. Bernasconi, and M. Parrinello, Phys. Rev. B 54, 9703 (1996).

${ }^{26}$ C. Mathioudakis, G. Kopidakis, P. Patsalas, and P. C. Kelires, Diamond Relat. Mater. 16, 1788 (2007)
${ }^{27}$ D. G. McCulloch, D. R. McKenzie, and C. M. Goringe, Phys. Rev. B 61, 2349 (2000)

${ }^{28}$ P. C. Kelires, Phys. Rev. Lett. 73, 2460 (1994).

${ }^{29}$ Y. Mo, M. Z. Bazant, and E. Kaxiras, Phys. Rev. B 70, 205210 (2004).

${ }^{30}$ M. M. J. Treacy and K. B. Borisenko, Science 335, 950 (2012).

${ }^{31}$ J. M. Soler, E. Artacho, J. D. Gale, A. García, J. Junquera, P. Ordejón, and D. Sánchez-Portal, J. Phys.: Condens. Matter 14, 2745 (2002).

${ }^{32}$ J. P. Perdew, A. Ruzsinszky, G. I. Csonka, O. A. Vydrov, G. E. Scuseria, L. A. Constantin, X. Zhou, and K. Burke, Phys. Rev. Lett. 100, 136406 (2008).

${ }^{33}$ J. Enkovaara, C. Rostgaard, J. J. Mortensen, J. Chen, M. Dułak, L. Ferrighi, J. Gavnholt, C. Glinsvad, V. Haikola, H. A. Hansen, H. H. Kristoffersen, M. Kuisma, A. H. Larsen, L. Lehtovaara, M. Ljungberg, O. LopezAcevedo, P. G. Moses, J. Ojanen, T. Olsen, V. Petzold, N. A. Romero, J. Stausholm-Møller, M. Strange, G. A. Tritsaris, M. Vanin, M. Walter, B. Hammer, H. Häkkinen, G. K. H. Madsen, R. M. Nieminen, J. K. Nørskov, M. Puska, T. T. Rantala, J. Schiøtz, K. S. Thygesen, and K. W. Jacobsen, J. Phys.: Condens. Matter 22, 253202 (2010).

${ }^{34}$ P. E. Blöchl, Phys. Rev. B 50, 17953 (1994).

${ }^{35}$ G. Onida, L. Reining, and A. Rubio, Rev. Mod. Phys. 74, 601 (2002).

${ }^{36}$ S. Sharma, J. K. Dewhurst, A. Sanna, and E. K. U. Gross, Phys. Rev. Lett. 107, 186401 (2011).

${ }^{37}$ S. Sharma, J. K. Dewhurst, A. Sanna, A. Rubio, and E. K. U. Gross, New J. Phys. 14, 053052 (2012).

${ }^{38}$ M. Kuisma, J. Ojanen, J. Enkovaara, and T. T. Rantala, Phys. Rev. B 82, 115106 (2010).

${ }^{39}$ O. Gritsenko, R. van Leeuwen, E. van Lenthe, and E. J. Baerends, Phys. Rev. A 51, 1944 (1995).

${ }^{40}$ J. Yan, K. W. Jacobsen, and K. S. Thygesen, Phys. Rev. B 84, 235430 (2011).

${ }^{41}$ A. Marini, R. Del Sole, and G. Onida, Phys. Rev. B 66, 115101 (2002).

${ }^{42}$ P. T. Jochym, K. Parlinski, and M. Sternik, Eur. Phys J. 10, 9 (1999).

${ }^{43}$ S. R. Bahn and K. W. Jacobsen, Comp. Sci. Eng. 4, 56 (2002).

${ }^{44}$ X. Yu, Y. Qin, C. B. Wang, Y. Q. Yang, and X. C. Ma, Vacuum 517, 4035-4038 (2009).

${ }^{45}$ H. Hirai, M. Terauchi, M. Tanaka, and K. Kondo, Phys. Rev. B 60, 6357 (1999).

${ }^{46}$ S. Logothetidis, Diamond Relat. Mater. 12, 141 (2003).

${ }^{47}$ H. Ehrenreich and H. R. Philipp, Phys. Rev. 128, 1622 (1962).

${ }^{48}$ J. R. Cole and N. J. Halas, Appl. Phys. Lett. 89, 153120 (2006). 